Article

\title{
Organic Extractives from Mentha spp. Honey and the Bee-Stomach: Methyl Syringate, Vomifoliol, Terpenediol I, Hotrienol and Other Compounds
}

\author{
Igor Jerković $^{1, *}$, Gordana Hegić ${ }^{2}$, Zvonimir Marijanović ${ }^{3}$ and Dragan Bubalo $^{2}$ \\ 1 Faculty of Chemistry and Technology, University of Split, N. Tesle 10/V, 21000 Split, Croatia \\ 2 Faculty of Agriculture, University of Zagreb, Svetošimunska 25, 10000 Zagreb, Croatia \\ 3 Marko Marulić Polytechnic in Knin, P. Krešimira IV 30, 22300 Knin, Croatia \\ * Author to whom correspondence should be addressed; E-Mail: igor@ktf-split.hr.
}

Received: 22 March 2010; in revised form: 13 April 2010 / Accepted: 20 April 2010 /

Published: 22 April 2010

\begin{abstract}
The GC and GC/MS analyses of the solvent organic extractive from the stomach of the bees, having collected Mentha spp. nectar, revealed the presence of methyl syringate $(6.6 \%)$, terpendiol I $(5.0 \%)$ and vomifoliol $(3.0 \%)$ that can be attributed to the plant origin. Other major compounds from the bee-stomach were related to the composition of cuticular waxes and less to pheromones. Organic extractives from Mentha spp. honey were obtained by solvent-free headspace solid-phase microextraction (HS-SPME) and ultrasonic solvent extraction (USE) and analyzed by GC and GC/MS. The major honey headspace compounds were hotrienol $(31.1 \%-38.5 \%)$, 2-methoxy-4-methylphenol $(0.5-6.0 \%)$, cisand trans-linalool oxides (0.9-2.8\%), linalool (1.0-3.1\%) and neroloxide (0.9-1.9\%). Methyl syringate was the most abundant compound (38.3-56.2\%) in the honey solvent extractives followed by vomifoliol (7.0-26.6\%). Comparison of the honey organic extractives with the corresponding bee-stomach extractive indicated that methyl syringate and vomofoliol were transferred to the honey while terpendiol I was partially transformed to hotrienol in ripened honey.
\end{abstract}

Keywords: Mentha spp. honey; bee-stomach; headspace solid-phase microextraction (HSSPME); ultrasonic solvent extraction (USE); gas chromatography and mass spectrometry (GC and GC/MS) 


\section{Introduction}

The chemical composition of the organic extractives is of great interest for characterizing the floral and/or geographical origin of honeys. In recent years a range of extractable natural organic substances that appear characteristic to be of the plant source have been identified as phytochemicals in different honeys [1,2]. For these analysis appropriate extraction methods should be applied to prevent the formation of artifacts generated by Strecker degradation, Maillard or non-enzymatic browning reactions [3]. In most cases, the extraction of organic compounds from the honey matrix is performed using solvents at room temperature [4,5], but the use of solvent-free systems (e.g. solid-phase microextraction) has also been proposed [6].

A broad range of aliphatic, aromatic, and/or degraded carotenoid-like structures have been reported in the honey extracts and headspace [1,2]. Unifloral honeys possess distinctive flavors, mainly derived from their nectar sources, indicating the presence of characteristic volatile components. The main components in source specific honey volatiles belong, in general, to three principal categories: terpenes, norisoprenoids, and benzene derivatives [1,2]. Some of these substances have been described as characteristics of the floral source, and other compounds, like some alcohols, branched aldehydes, and furan derivatives, may be related to the microbial purity of honey processing and storage conditions. Specific markers of the botanical origin were also found such as methyl anthranilate for citrus honey [7], trans-oak lactone for holm-oak honeydew [8] or 2-amino- and 4-aminoacetophenone for chestnut honey [9].

Mentha, the genus of Lamiaceae family, includes 25 species that are spread all over the world. Among them, Mentha pulegium L. and Mentha aquatica L., are well known aromatic plants. The constituents of $M$. pulegium L. oil have been studied [10,11] and a difference in the composition of the oil major components (pulegone, isomenthone and piperitone) depending on the region of cultivation in different countries have been found. The essential oil of $M$. aquatica L. is characterized by domination of menthofuran [12]. In general, very few references can be found on Mentha spp. honeys $[13,14]$, but without any chemical composition data. As Lamiaceae pollen is under-represented in the spectra, the minimum pollen percentage for Mentha spp. honeys has been established at 20\% [15,16].

There are only a few studies in which the organic extractives of the bee-stomach have been correlated with those of the corresponding honey. Most recently, the comparison of the components of the extracts of linden honey and bee-stomach showed that nectar and honey stomach contain many aldehydes which were found as corresponding acids in the honey [17]. To the best of our knowledge, organic extractives from Mentha spp. honey and from the corresponding bee-stomach have not yet been studied. A two-way approach was used for the research of honey volatiles: solvent-free headspace solid-phase microextraction (HS-SPME) and ultrasonic solvent extraction (USE). Isolated organic extractives were analyzed by gas chromatography and mass spectrometry (GC and GC/MS).

\section{Results and Discussion}

The gathered nectar is stocked in the bee-stomach, which can contain up to $60 \mu \mathrm{L}$ of liquid, and the honey sacs containing Mentha spp. pollen grains were investigated. The enzymes in the salvia degrade nectar sucrose into glucose and fructose and cleave the glycosides. On returning to the hive, the 
content of the stomach is regurgitated into the waxy honeycomb and is ripened into the honey. Under the highly oxidative atmosphere of the honeycomb sensitive honey organic compounds can undergo oxidation [17]. The content of the stomach of the bees caught at the entrance of the hive on their way back from Mentha spp. nectar gathering was isolated by USE and compared to the extractives obtained from the ripe honey with focus on determination of plant derived compounds. The unifloral botanical origin of all the honey samples was confirmed by pollen analysis (up to 49\% of Mentha spp. pollen dominated by Mentha aquatica L. and Mentha pulegium L.) according to recommended minimal values $[15,16]$. Identified accompanying pollen grains were from Amorpha fruticosa, Centaurea spp., Asteraceae and Brassicaceae with minor percentages of pollen from other species.

The results presented below indicate great variability of the honey organic extractives composition strongly depending on the isolation method. Solvent-free HS-SPME method selectively isolated the honey headspace organic extractives dominated by high volatile compounds that were present with minor percentages in the honey solvent extracts. The medium-low volatile and relatively high polar organic compounds were the most abundant in the solvent extracts with limitations observed in the headspace composition. Both organic extractives were comprehensive and reliable for characterization of this honey without the presence of thermal derived artifacts.

\subsection{Organic Extractives Isolated by Ultrasonic Solvent Extraction from the Bee-Stomach and Honey}

The content of the stomach of 70 bees caught at the entrance of the hive on their way back from Mentha spp. nectar gathering was isolated by USE and analyzed (a representative chromatogram is presented in Figure 1). The bee-stomach organic extract was mainly composed of the following groups of natural compounds (Table 1): fatty acids [(Z)-octadec-9-enoic acid (30.4\%), hexadecanoic acid $(6.4 \%)]$, fatty alcohols [(Z)-octadec-9-en-1-ol (5.7\%), hexadecan-1-ol (3.3\%), octadecan-1-ol (1.9\%)] as well as higher aliphatic hydrocarbons [tetracosane $(9.0 \%)$, heneicosane $(0.7 \%)$ ]. These chemical structures are particularly related to the composition of cuticular waxes and less to pheromones. The cuticular waxes contain aliphatic compounds from $\mathrm{C}_{18}$ to $\mathrm{C}_{54}$ dominated by hydrocarbons [18]. "Queen's pheromone", a well-equilibrated cocktail of fatty acids and aromatic compounds, is one of the most important sets of pheromones in the bee hive [19]. Only several structurally-related compounds of queen retinue pheromone were found in bee-stomach such as hexadecan-1-ol and different fatty acids. The major identified terpene in the bee-stomach was 3,7-dimethylocta-1,5-dien3,7-diol, terpendiol I (5.0\%) originated probably by glycosidase activity (cleavage of the nectar glycosides) in the salvia. This activity was already confirmed by the appearance of new monoterpenic alcohols (three linalool derivatives: 3,7-dimethylocta-1,5-dien-3,7-diol, 3,7-dimethylocta-1,6-dien-3,5diol and 2,6-dimethyl-6-hydroxyocta-2,7-dienal) in the extract of the liquid isolated from the bee stomach having collected linden nectar [17]. Monoterpenic alcohol 3,7-dimethylocta-1,5-dien-3,7-diol, terpendiol I was found in Mentha spp. honey solvent extracts, but also can transform to hotrienol, the most abundant compound in the honey headspace, Table 2. Among all identified compounds, methyl syringate $(6.6 \%)$ and vomifoliol $(3.0 \%)$ can be considered plant derived phytochemicals in the beestomach having collected nectar from Mentha spp. flowers. 
Figure 1. Representative TIC chromatogram of the bee-stomach organic extractive.

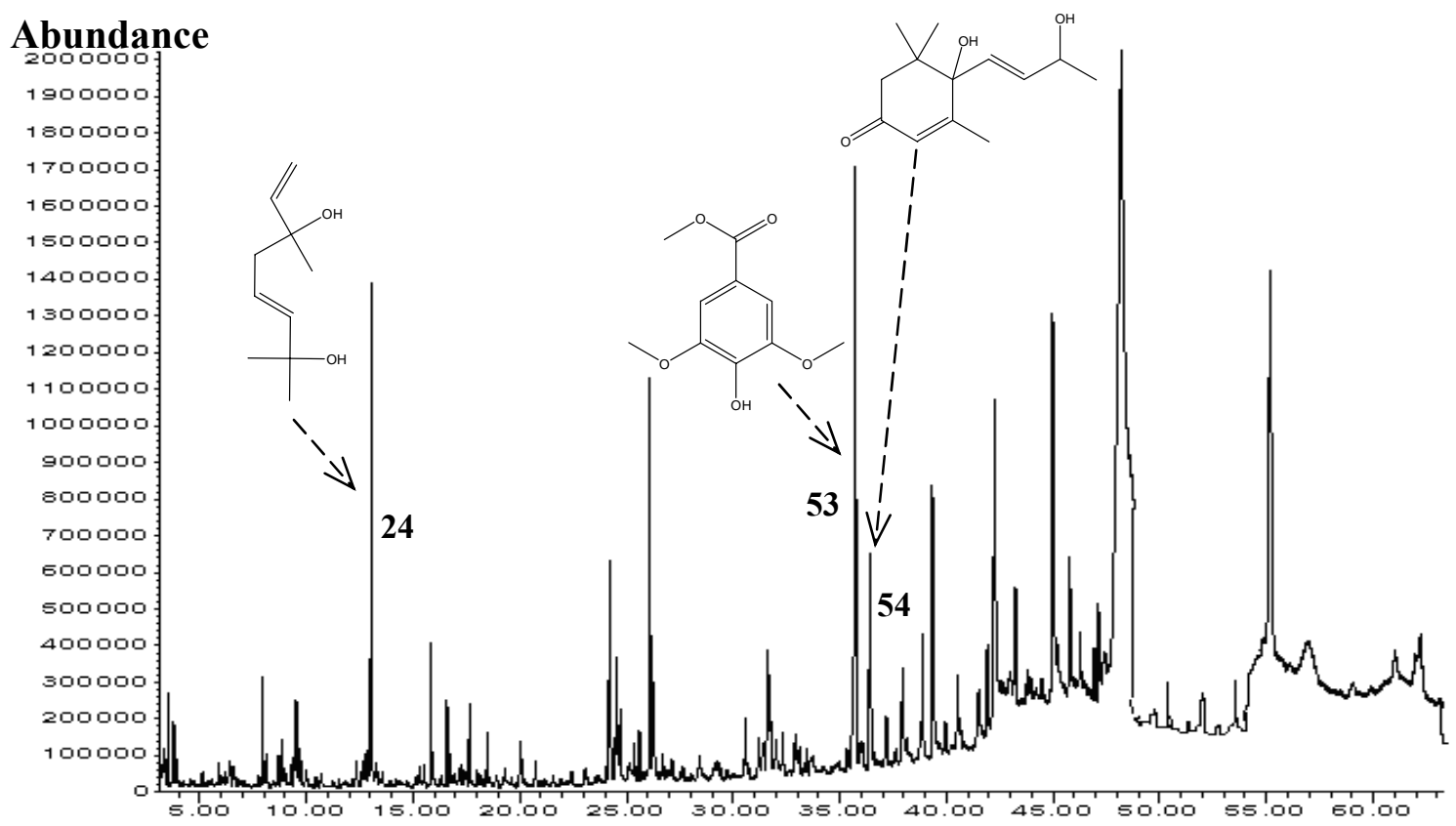

Time/min

Table 1. Organic extractives composition from Mentha spp. honey and the bee-stomach.

\begin{tabular}{|c|c|c|c|c|c|c|c|c|c|c|c|}
\hline \multirow{3}{*}{ No. } & \multirow{3}{*}{ Compound } & \multirow{3}{*}{ RI } & \multicolumn{9}{|c|}{ Area percentage $(\%)$} \\
\hline & & & \multicolumn{4}{|c|}{$\mathbf{A}$} & \multicolumn{4}{|c|}{ B } & \multirow[t]{2}{*}{$\mathrm{C}$} \\
\hline & & & Min. & Max. & Av. & SD. & Min. & Max. & Av. & SD. & \\
\hline 1. & $\begin{array}{l}\text { 2-Methylbutanoic } \\
\text { acid }\end{array}$ & $<900$ & 0.0 & 0.1 & 0.07 & 0.06 & - & - & - & - & - \\
\hline 2. & 2-Furanmethanol & $<900$ & 0.1 & 0.2 & 0.13 & 0.06 & 0.0 & 0.2 & 0.10 & 0.10 & - \\
\hline 3. & 4-Methyloctane & $<900$ & - & - & - & - & - & - & - & - & 0.2 \\
\hline 4. & 1,4-Dimethylbenzene & $<900$ & 0.0 & 0.1 & 0.07 & 0.06 & - & - & - & - & 0.6 \\
\hline 5. & Heptan-2-one & $<900$ & - & - & - & - & - & - & - & - & 0.3 \\
\hline 6. & $\begin{array}{l}\text { 1-(2-Furanyl)- } \\
\text { ethanone }\end{array}$ & 914 & 0.0 & 0.1 & 0.03 & 0.06 & - & - & - & - & - \\
\hline 7. & Benzaldehyde $^{\mathrm{a}}$ & 965 & 0.0 & 0.1 & 0.03 & 0.06 & - & - & - & - & - \\
\hline 8. & (Z)-Hex-3-enoic acid & 1013 & 0.1 & 0.3 & 0.20 & 0.10 & - & - & - & - & - \\
\hline 9. & Limonene $^{\mathrm{a}}$ & 1036 & 0.0 & 0.1 & 0.03 & 0.06 & - & - & - & - & - \\
\hline 10. & Benzyl alcohol $^{\mathrm{a}}$ & 1037 & 0.1 & 0.5 & 0.30 & 0.20 & 0.0 & 0.5 & 0.27 & 0.25 & - \\
\hline 11. & Phenylacetaldehyde ${ }^{a}$ & 1048 & 0.0 & 0.1 & 0.07 & 0.06 & - & - & - & - & - \\
\hline 12. & 5-Methylundecane ${ }^{*}$ & 1067 & - & - & - & - & - & - & - & - & 0.8 \\
\hline 13. & $\begin{array}{l}\text { trans-Linalool oxide } \\
\text { (furan type) }\end{array}$ & 1076 & 0.0 & 0.1 & 0.07 & 0.06 & - & - & - & - & - \\
\hline 14. & $\begin{array}{l}\text { 4,5-Dimethyl-2- } \\
\text { formylfuran }\end{array}$ & 1078 & 0.0 & 0.2 & 0.10 & 0.10 & 0.0 & 0.2 & 0.10 & 0.10 & - \\
\hline 15. & Methyl 2-furoate & 1084 & 0.1 & 0.7 & 0.47 & 0.32 & 0.2 & 0.9 & 0.63 & 0.38 & - \\
\hline 16. & Undecane $^{\mathrm{a}}$ & 1100 & - & - & - & - & - & - & - & - & 0.2 \\
\hline 17. & Hotrienol & 1106 & 0.2 & 0.6 & 0.47 & 0.23 & 0.1 & 0.6 & 0.43 & 0.29 & - \\
\hline
\end{tabular}


Table 1. Cont.

\begin{tabular}{|c|c|c|c|c|c|c|c|c|c|c|c|}
\hline 18. & 2-Phenylethanol ${ }^{\mathrm{a}}$ & 1116 & 0.1 & 0.4 & 0.30 & 0.17 & 0.2 & 0.4 & 0.33 & 0.11 & - \\
\hline 19. & $\begin{array}{l}\text { 5-Hydroxy-2-methyl- } \\
\text { 4H-Pyran-4-one }\end{array}$ & 1139 & 0.0 & 0.2 & 0.10 & 0.10 & 0.0 & 0.1 & 0.07 & 0.06 & - \\
\hline 20. & $\begin{array}{l}\text { 2,3-Dihydro-3,5- } \\
\text { dihydroxy-6-methyl- } \\
\text { 4H-pyran-4-one }\end{array}$ & 1145 & 0.0 & 0.2 & 0.10 & 0.10 & 0.0 & 0.2 & 0.13 & 0.11 & - \\
\hline 21. & Octanoic acid & 1174 & - & - & - & - & - & - & - & - & 0.4 \\
\hline 22. & Benzoic acid $^{\mathrm{a}}$ & 1162 & 0.4 & 1.3 & 0.97 & 0.49 & 0.2 & 0.9 & 0.63 & 0.38 & - \\
\hline 23. & $\begin{array}{l}\text { 3,5-Dihydroxy-2- } \\
\text { methyl- } \\
\text { 4H-pyran-4-one }\end{array}$ & 1189 & 0.0 & 0.2 & 0.10 & 0.10 & - & - & - & - & - \\
\hline 24. & $\begin{array}{l}\text { 3,7-Dimethylocta- } \\
\text { 1,5-dien-3,7-diol } \\
\text { (terpendiol I) }\end{array}$ & 1191 & 1.0 & 4.9 & 3.17 & 1.98 & 0.7 & 2.5 & 1.57 & 0.90 & 5.0 \\
\hline 25. & Dodecane $^{\mathrm{a}}$ & 1200 & - & - & - & - & - & - & - & - & 0.8 \\
\hline 26. & $\begin{array}{l}\text { 5-Hydroxymethyl- } \\
\text { furfural }\end{array}$ & 1230 & 0.1 & 2.7 & 1.60 & 1.35 & 0.2 & 5.1 & 2.97 & 2.51 & - \\
\hline 27. & $(E)$-Dec-2-enal & 1268 & - & - & - & - & - & - & - & - & 1.2 \\
\hline 28. & Phenylacetic acid ${ }^{\mathrm{a}}$ & 1269 & 1.0 & 7.7 & 3.83 & 3.44 & 0.3 & 5.1 & 2.37 & 2.47 & - \\
\hline 29. & Nonanoic acid ${ }^{\mathrm{a}}$ & 1273 & - & - & - & - & - & - & - & - & 0.3 \\
\hline 30. & 4-Acetylanilline & 1308 & 0.0 & 0.2 & 0.10 & 0.10 & - & - & - & - & - \\
\hline 31. & $\begin{array}{l}\text { 3-Hydroxy-4-phenyl- } \\
\text { butan-2-one }\end{array}$ & 1354 & 0.8 & 1.9 & 1.33 & 0.55 & 0.6 & 1.2 & 0.83 & 0.32 & - \\
\hline 32. & $\begin{array}{l}\text { Phenylpropanoic } \\
\text { acid }^{\mathrm{a}}\end{array}$ & 1361 & 0.0 & 0.6 & 0.27 & 0.31 & 0.0 & 0.3 & 0.17 & 0.15 & - \\
\hline 33. & 1-Hydroxylinalool & 1365 & 0.1 & 0.4 & 0.23 & 0.15 & 0.2 & 0.3 & 0.20 & 0.10 & - \\
\hline 34. & 2-Phenylacetamide & 1393 & 0.0 & 0.1 & 0.07 & 0.06 & 0.0 & 0.5 & 0.23 & 0.25 & - \\
\hline 35. & 2-Aminobenzoic acid & 1416 & 0.0 & 0.8 & 0.33 & 0.42 & 0.0 & 0.7 & 0.27 & 0.38 & - \\
\hline 36. & $\begin{array}{l}\text { 4-Hydroxybenzyl } \\
\text { alcohol } \\
\text { 2,6-bis }(1,1-\end{array}$ & 1426 & 0.1 & 0.2 & 0.17 & 0.06 & - & - & - & - & - \\
\hline 37. & $\begin{array}{l}\text { Dimethylethyl)- } \\
\text { cyclohexa-2,5-diene- } \\
\text { 1,4-dione }\end{array}$ & 1473 & - & & - & - & - & - & - & - & 2.3 \\
\hline 38. & $\begin{array}{l}\text { 2,6-di(1,1- } \\
\text { Dimethylethyl)-4- } \\
\text { hydroxy-4-methyl- } \\
\text { cyclohexa-2,5-dien- } \\
\text { 1-one }\end{array}$ & 1841 & - & - & - & - & - & - & - & - & 1.3 \\
\hline 39. & $\begin{array}{l}\text { 2,6-di(1,1- } \\
\text { Dimethylethyl)-4- } \\
\text { metylene-cyclohexa- } \\
\text { 2,5-diene-1-one }\end{array}$ & 1845 & - & - & - & - & - & - & - & - & 1.0 \\
\hline 40. & Acetylvanillone & 1494 & 0.0 & 0.3 & 0.17 & 0.15 & 0.0 & 0.3 & 0.17 & 0.15 & - \\
\hline 41. & Pentadecane ${ }^{a}$ & 1500 & - & - & - & - & - & - & - & - & 0.3 \\
\hline 42. & $\begin{array}{l}\text { 2,4-bis(1,1- } \\
\text { Dimethylethyl)phenol }\end{array}$ & 1525 & - & - & - & - & - & - & - & - & 1.2 \\
\hline 43. & Methyl vanillate $^{\mathrm{a}}$ & 1527 & 0.4 & 1.3 & 0.83 & 0.45 & 0.3 & 0.8 & 0.60 & 0.26 & - \\
\hline 44. & $\begin{array}{l}\text { 4-Hydroxybenzoic } \\
\text { acid }^{\mathrm{a}}\end{array}$ & 1558 & 0.3 & 0.9 & 0.67 & 0.32 & 0.0 & 0.2 & 0.10 & 0.10 & - \\
\hline 45. & Vanillic acid ${ }^{\mathrm{a}}$ & 1566 & 0.2 & 0.8 & 0.50 & 0.30 & 0.1 & 0.3 & 0.20 & 0.10 & - \\
\hline 46. & $\begin{array}{l}\text { 3-Hydroxy- } \beta \text { - } \\
\text { damascone }\end{array}$ & 1617 & 0.0 & 0.5 & 0.27 & 0.25 & 0.1 & 0.3 & 0.20 & 0.10 & - \\
\hline 47. & $\begin{array}{l}\text { Isopropyl- } \\
\text { pseudocumene }\end{array}$ & 1657 & 0.3 & 1.8 & 1.13 & 0.76 & 0.5 & 1.7 & 1.07 & 0.60 & 0.6 \\
\hline
\end{tabular}


Table 1. Cont.

\begin{tabular}{|c|c|c|c|c|c|c|c|c|c|c|c|}
\hline 48. & Menthofuran & 1661 & 0.0 & 0.4 & 0.20 & 0.20 & 0.0 & 0.4 & 0.17 & 0.21 & - \\
\hline 49. & Syringaldehyde & 1662 & 0.0 & 0.1 & 0.07 & 0.06 & 0.0 & 0.2 & 0.10 & 0.10 & - \\
\hline 50. & $\begin{array}{l}\text { Isopropyl- } \\
\text { pseudocumene }^{* *}\end{array}$ & 1678 & 0.0 & 1.8 & 1.03 & 0.930 & 0.5 & 1.7 & 1.07 & 0.60 & - \\
\hline 51. & 8-Hydroxyquinoline & 1720 & - & - & - & - & 0.4 & 1.0 & 0.63 & 0.32 & - \\
\hline 52. & $\begin{array}{l}\text { 4-Ethybenzen-1,3- } \\
\text { diol }\end{array}$ & 1743 & 0.3 & 0.4 & 0.37 & 0.06 & 0.2 & 0.7 & 0.40 & 0.26 & - \\
\hline 53. & Methyl syringate $^{a}$ & 1744 & 47.9 & 56.2 & 52.30 & 4.17 & 38.3 & 48.6 & 43.57 & 5.15 & 6.6 \\
\hline 54. & Vomifoliol & 1802 & 7.0 & 10.3 & 8.27 & 1.78 & 16.9 & 26.6 & 20.43 & 5.36 & 3.0 \\
\hline 55. & 3-Ethylbenzophenone & 1826 & - & - & - & - & - & - & - & - & 0.6 \\
\hline 56. & $\begin{array}{l}\text { 3-(4-Hydroxy- } \\
\text { phenyl)-prop-2-enoic } \\
\text { acid }\end{array}$ & 1831 & 0.4 & 0.8 & 0.67 & 0.23 & - & - & - & - & - \\
\hline 57. & Neophytadiene & 1849 & - & - & - & - & - & - & - & - & 1.5 \\
\hline 58. & Ferulic acid ${ }^{a}$ & 1867 & 0.0 & 0.2 & 0.10 & 0.10 & - & - & - & - & - \\
\hline 59. & Diisobuthyl phtalate & 1869 & 0.1 & 0.2 & 0.13 & 0.06 & 0.2 & 0.3 & 0.23 & 0.06 & 1.5 \\
\hline 60. & Hexadecan-1-ol ${ }^{\mathrm{a}}$ & 1882 & 0.5 & 1.0 & 0.70 & 0.26 & 0.9 & 1.2 & 1.03 & 0.15 & 3.3 \\
\hline 61. & Nonadecane $^{\mathrm{a}}$ & 1900 & - & - & - & - & - & - & - & - & 0.3 \\
\hline 62. & Hexadecanoic acid ${ }^{a}$ & 1963 & 0.8 & 6.7 & 2.97 & 3.25 & 0.7 & 1.8 & 1.30 & 0.56 & 6.4 \\
\hline 63. & $\begin{array}{l}\text { (Z)-Octadec-9-en-1- } \\
\mathrm{ol}^{\mathrm{a}}\end{array}$ & 2060 & 1.0 & 1.6 & 1.27 & 0.31 & 1.3 & 2.4 & 1.93 & 0.57 & 5.7 \\
\hline 64. & Octadecan-1-ol ${ }^{\mathrm{a}}$ & 2084 & 0.2 & 0.3 & 0.27 & 0.06 & 0.1 & 0.6 & 0.37 & 0.25 & 1.9 \\
\hline 65. & Heneicosane $^{a}$ & 2100 & - & - & - & - & - & - & - & - & 0.7 \\
\hline 66. & $\begin{array}{l}\text { (Z)-Octadec-9-enoic } \\
\text { acid }^{\mathrm{a}}\end{array}$ & 2147 & 0.3 & 1.5 & 0.80 & 0.62 & 0.6 & 2.0 & 1.13 & 0.76 & 30.4 \\
\hline 67. & (Z)-Tricos-9-ene & 2330 & - & - & - & - & - & - & - & - & 2.2 \\
\hline 68. & Tetracosane $^{a}$ & 2400 & 0.7 & 2.4 & 1.30 & 0.95 & 0.8 & 2.0 & 1.50 & 0.62 & 9.0 \\
\hline
\end{tabular}

$\mathrm{A}=$ Mentha spp. honey solvent extract with pentane and diethyl ether $(1: 2, \mathrm{v} / \mathrm{v}) ; \mathrm{B}=$ Mentha spp. honey solvent extract with dichloromethane; $\mathrm{C}$ - bee-stomach solvent extract with dichloromethane; $\mathrm{RI}=$ retention indices on HP-5MS column; - = not identified; Min. = minimal percentage; Max. = maximal percentage; Av. $=$ average percentage; SD.$=$ standard deviation; ${ }^{\mathrm{a}}$ - identification confirmed with reference compound; ${ }^{*}$ tentatively identified; ${ }^{* *}$ - correct isomer not identified.

Methyl syringate was the most abundant compound of all Mentha ssp. honey solvent extracts (Table 1, Figure 2) with minor percentage differences among different solvents: 47.9-56.2\% (solvent pentanediethyl ether $1: 2 \mathrm{v} / \mathrm{v}$ ); 38.3-48.6\% (solvent dichloromethane). Methyl syringate was detected also in robinia, rape, chestnut, clover, linden blossom, dandelion, sunflower, thyme, manuka and fir honeys [20], but only in asphodel honey it reached the highest level [21]. Benzoic acid derivatives can be produced through the shikimate pathway [22], and methyl syringate [23,24] and its glucoside [25] were found in several plant extracts, but syringic acid methyl ester is also a typical laccase substrate in mediated breakdown of lignocellulose. Although methyl syrigate was considered previously [17] to be probably absorbed into the lipophilic honey from propolis of linden honey, preliminary research of the propolis composition gathered from the combs containing Mentha spp. honey (unpublished data) confirmed its absence in the collected propolis. In addition, it was not identified among the volatiles of propolis gathered from different region in Croatia [26]. Since methyl syringate was found in the corresponding bee-stomach extractive, it can be considered as phytochemical transferred to the honey. Among other shikimate pathway derivatives found (Table 1) the following compounds were the most 
abundant: phenylacetic acid (0.3-7.7\%), methyl vanillate $(0.3-1.3 \%)$, vanillic acid $(0.1-0.8 \%)$ and 4hydroxybenzoic acid $(0.0-0.9 \%)$. However, in comparison with other unifloral honeys neither of the previous aromatic acids can be emphasized as potential marker of the botanical origin. Vomifoliol was the second abundant compound in the solvent extracts (7.0-26.6\%), the most widespread 3,5,5trimethylcyclohex-2-ene derivative (carotenoid degraded-like structure) in the honey samples. This compound probably arises through degradation of abscissic acid, a well-known growth hormone. The origin of degraded carotenoid-like structures in different honeys was already connected with the corresponding botanical sources [27]. In addition, vomifoliol was found in the extractive of beestomach. Another single abundant compound in the honey extracts was 3-hydroxy-4-phenyl-butan-2one $(0.6-1.9 \%)$. Furan derivatives were also found with minor percentages [5-hydroxymethylfurfural $(0.1-5.1 \%)$, 2-furanmethanol $(0.0-0.2 \%)$ and methyl 2-furoate $(0.1-0.9 \%)]$, not as the honey markers but as indicators of absence of heat treatment and appropriate storage conditions.

Figure 2. Representative TIC chromatogram of organic extractives obtained with the mixture of pentane and diethyl ether $(1: 2, \mathrm{v} / \mathrm{v})$ of Mentha spp. honey.

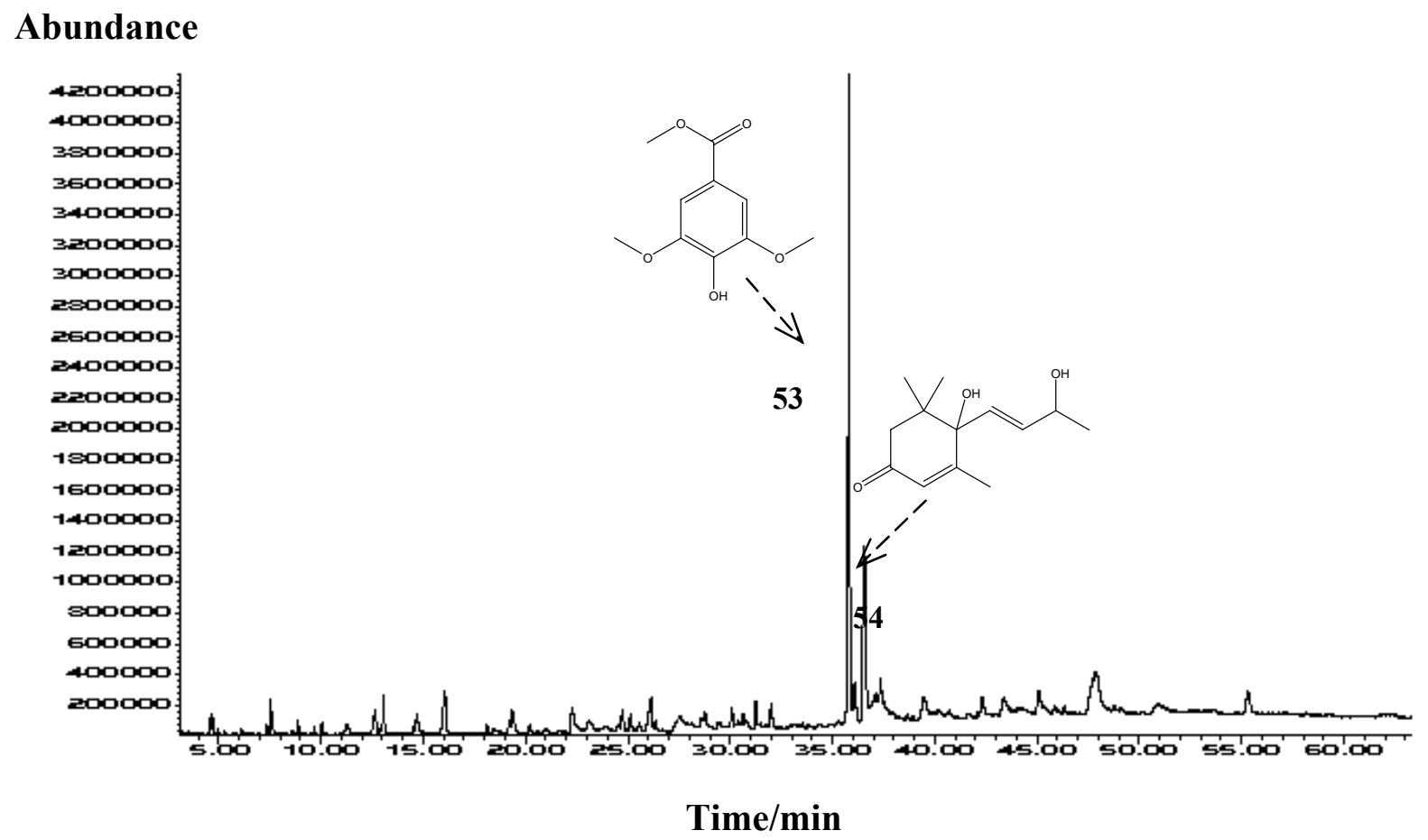

Besides methyl syringate, vomifoliol and 3,7-dimethylocta-1,5-dien-3,7-diol common compounds found in the honey and the bee-stomach were fatty acids, alcohols and carbonyls. Their presence was expected, not only because they are present in the bee-stomach, but also in the beeswax and therefore they cannot be considered important for botanical origin determination [28]. 


\subsection{Organic Extractives Isolated by Headspace Solid-Phase Microextraction from the Honey}

Terpenes were the most abundant compounds of Mentha spp. honey headspace (Table 2). The origin of terpenes and derivatives in the honey is usually associated to the floral nectar gathered by the honeybees.

Table 2. Mentha spp. honey solvent-free organic extractives composition isolated by HS-SPME.

\begin{tabular}{|c|c|c|c|c|c|c|}
\hline \multirow{2}{*}{ No. } & \multirow{2}{*}{ Compound } & \multirow{2}{*}{ RI } & \multicolumn{4}{|c|}{ Area percentage (\%) } \\
\hline & & & Min. & Max. & Av. & SD. \\
\hline 1. & 2-Furancarboxaldehyde & $<900$ & 0.0 & 2.1 & 1.07 & 1.05 \\
\hline 2. & 3-Methylbutanoic acid & $<900$ & 0.0 & 1.4 & 0.57 & 0.74 \\
\hline 3. & 2,5-Diethyltetrahydrofuran ${ }^{*}$ & 902 & 0.0 & 2.0 & 0.97 & 1.00 \\
\hline 4. & 1-(2-Furanyl)-ethanone & 914 & 0.0 & 0.4 & 0.23 & 0.21 \\
\hline 5. & Benzaldehyde $^{a}$ & 965 & 0.0 & 0.8 & 0.47 & 0.42 \\
\hline 6. & Hexanoic acid ${ }^{\mathrm{a}}$ & 974 & 0.0 & 0.3 & 0.13 & 0.15 \\
\hline 7. & (Z)-Hex-3-enoic acid & 1013 & 0.0 & 1.0 & 0.43 & 0.51 \\
\hline 8. & Benzyl alcohol $^{\mathrm{a}}$ & 1037 & 0.7 & 1.0 & 0.83 & 0.15 \\
\hline 9. & Phenylacetaldehyde ${ }^{a}$ & 1048 & 0.8 & 1.3 & 1.07 & 0.25 \\
\hline 10. & trans-Linalool oxide (furan type) & 1076 & 1.8 & 2.8 & 2.37 & 0.51 \\
\hline 11. & cis-Linalool oxide (furan type) & 1091 & 0.9 & 1.9 & 1.43 & 0.50 \\
\hline 12. & Linalool $^{\mathrm{a}}$ & 1101 & 1.0 & 3.1 & 1.80 & 1.14 \\
\hline 13. & Hotrienol & 1106 & 31.1 & 38.5 & 33.90 & 4.01 \\
\hline 14. & 2-Phenylethanol ${ }^{\mathrm{a}}$ & 1116 & 1.0 & 2.8 & 1.73 & 0.95 \\
\hline 15. & $\alpha$-Isophorone & 1124 & 0.0 & 0.6 & 0.27 & 0.31 \\
\hline 16. & $p$-Mentha-1,5,8-triene ${ }^{* *}$ & 1138 & 0.0 & 0.5 & 0.23 & 0.25 \\
\hline 17. & Lilac aldehyde (isomer I) ${ }^{* *}$ & 1159 & 0.0 & 0.5 & 0.23 & 0.25 \\
\hline 18. & Neroloxide & 1162 & 0.9 & 1.9 & 1.37 & 0.50 \\
\hline 19. & Epoxylinalool & 1178 & 0.0 & 0.3 & 0.13 & 0.15 \\
\hline 20. & Benzoic acid $^{\mathrm{a}}$ & 1162 & 0.0 & 0.9 & 0.40 & 0.46 \\
\hline 21. & Octanoic acid ${ }^{\mathrm{a}}$ & 1174 & 0.0 & 1.6 & 0.63 & 0.85 \\
\hline 22. & 3,7-Dimethylocta-1,5-dien-3,7-diol & 1191 & 0.0 & 4.7 & 2.13 & 2.38 \\
\hline 23. & 2-Methoxy-4-methylphenol & 1199 & 0.5 & 6.0 & 2.73 & 2.89 \\
\hline 24. & 5-Hydroxymethylfurfural ${ }^{\mathrm{a}}$ & 1230 & 0.0 & 1.0 & 0.53 & 0.50 \\
\hline 25. & Phenylacetic acid ${ }^{\mathrm{a}}$ & 1269 & 0.0 & 0.8 & 0.47 & 0.42 \\
\hline 26. & Nonanoic acid ${ }^{\mathrm{a}}$ & 1273 & 0.0 & 2.0 & 0.93 & 1.00 \\
\hline 27. & 2-Acetylanilline & 1308 & 0.0 & 2.6 & 1.13 & 1.33 \\
\hline 28. & 4-Vinyl-2-methoxyphenol & 1322 & 0.0 & 0.9 & 0.57 & 0.49 \\
\hline 29. & 3-Hydroxy-4-phenyl-butan-2-one & 1354 & 1.0 & 2.5 & 1.63 & 0.78 \\
\hline 30. & cis-Isoeugenol & 1366 & 0.0 & 0.5 & 0.20 & 0.26 \\
\hline 31. & Decanoic acid ${ }^{\mathrm{a}}$ & 1370 & 0.0 & 0.5 & 0.20 & 0.26 \\
\hline 32. & trans- $\beta$-Damascenone & 1388 & 0.0 & 1.5 & 0.67 & 0.76 \\
\hline 33. & Methyl vanillate ${ }^{\mathrm{a}}$ & 1527 & 0.0 & 0.9 & 0.47 & 0.45 \\
\hline 34. & Methyl syringate ${ }^{a}$ & 1744 & 1.0 & 2.4 & 1.87 & 0.76 \\
\hline 35. & Nonadecane $^{a}$ & 1900 & 0.0 & 0.6 & 0.27 & 0.31 \\
\hline 36. & Hexadecanoic acid ${ }^{\mathrm{a}}$ & 1963 & 1.0 & 2.1 & 1.60 & 0.56 \\
\hline
\end{tabular}

$\mathrm{RI}=$ retention indices on HP-5MS column; - = not identified; Min. = minimal percentage; Max. $=$ maximal percentage; Av. $=$ average percentage; SD. $=$ standard deviation; ${ }^{\mathrm{a}}-$ identification confirmed with reference compound; ${ }^{*}$ - tentatively identified; ${ }^{* *}$ - correct isomer not identified. 
The major honey headspace compound was hotrienol $(31.1 \%-38.5 \%)$ followed by cis- and translinalool oxides (0.9-2.8\%), linalool (1.0-3.1\%) and neroloxide (0.9-1.9\%). A representative chromatogram of solvent-free organic extractives obtained by HS-SPME is presented in Figure 3. Hotrienol is known to be a thermally generated product, but the honey samples were not heated and therefore found hotrienol cannot be considered a thermally generated artifact in this case. These findings support a natural occurrence of hotrienol in non-thermally treated honey. Much lower proportions of hotrienol were found in unripe than in ripe honey, suggesting that hotrienol is probably formed during honey ripening [29] within the hive conditions (temperature, $\mathrm{pH}$, enzymes that can lead to the oxidative degradation of linalool or the cleavage of the glycosidic bonds). Hotrienol can be derived either from 2,6-dimethyl-3,7-octadiene-2,6-diol (or its glycoconjugate form), from the allylic rearrangement of 3,7-dimethyl-1,7-octadiene-3,6-diol, or from dehydratation of 8-hydroxylinalool [30].

Figure 3. Representative TIC chromatogram of solvent-free extractives of Mentha spp. honey obtained by HS-SPME.

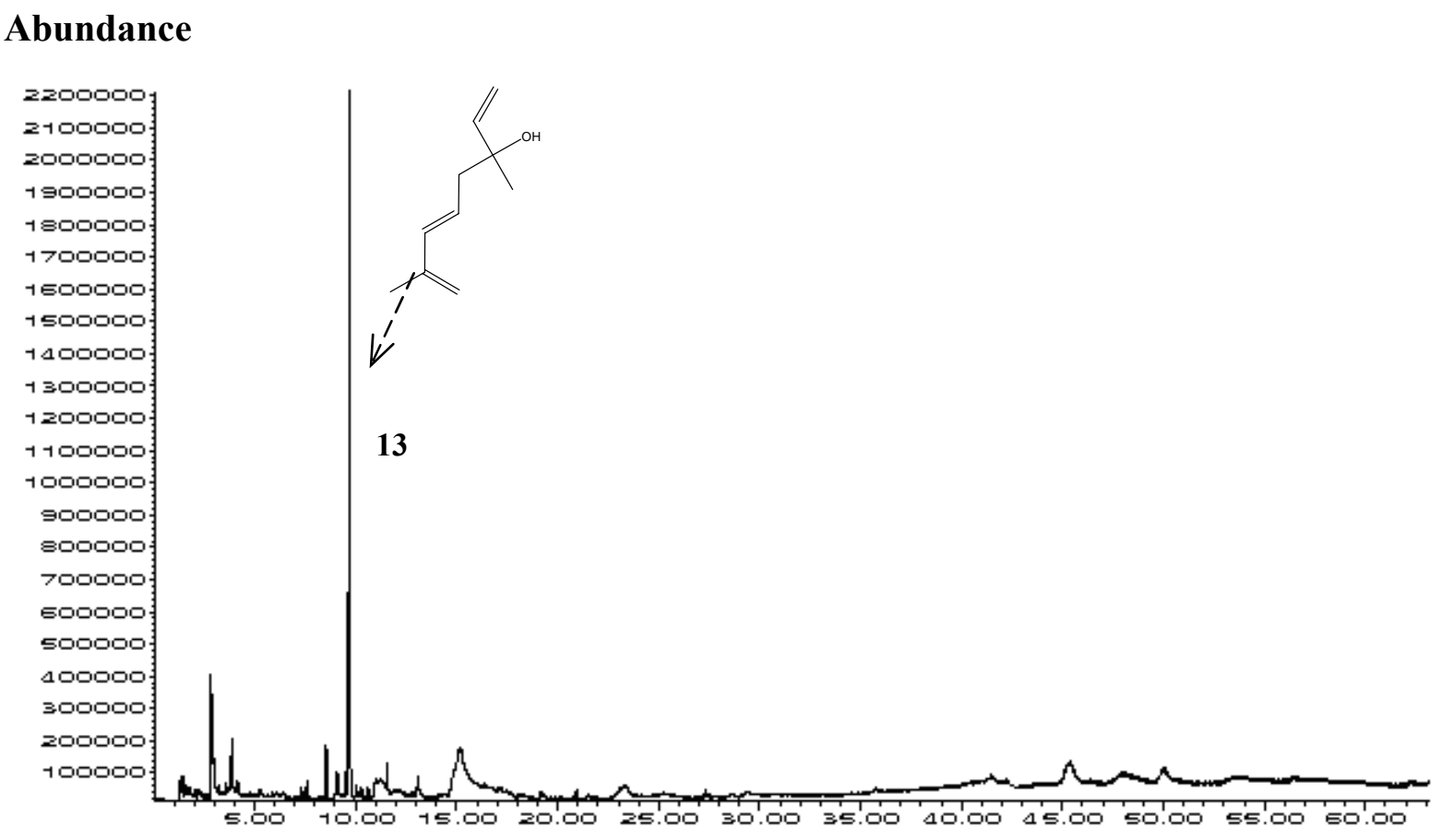

Time/min

Hotrienol has been reported as a constituent of different honeys and therefore cannot be considered specific for Mentha spp. honey. However its high percentage in the headspace is a notable characteristic of this particular honey. Hotrienol, although present in honeys of various floral origins has been markedly detected in lavander honeys [31]. Benzaldehyde (0.0-0.8\%), benzyl alcohol (0.7-1.0\%), phenylacetaldehyde $(0.8-1.3 \%)$, 2-phenylethanol (1.0-2.8\%) and 3-hydroxy-4phenylbutan-2-one (1.0-2.5\%) were the most abundant benzene derivatives in the headspace. Methyl syringate (1.0-2.4\%) and hexadecanoic acid (1.0-2.1\%) were found with low percentages, as was expected. 


\section{Experimental}

\subsection{Honey Samples and Bee-Stomach}

Six Mentha spp. honey samples were investigated. The samples were obtained from professional beekeepers and no mechanical treatment or heat was used. The combs were placed in the area of wild growing Mentha spp. dominated by Mentha aquatica L. and Mentha pulegium L. Melissopalynological analysis was performed by the methods recommended by the International Commission for Bee Botany [32]. Microscopical examination was carried out on a Hund h 500 (Wetzlar, Germany) light microscope attached to a digital camera (Motic m 1000) and coupled to an image analysis system (Motic Images Plus software) for morphometry of pollen grains. Each sample was examined to determine the percentage of pollen grains. All the samples were stored in hermetically closed glass bottles at $4{ }^{\circ} \mathrm{C}$ until the volatiles isolation.

During the Mentha spp. honey flow the samples of returning foragers were collected. The bees were frozen in the field by liquid nitrogen and were stored in deep-freezer until their honey-sac contents was analyzed. After thawing, the abdomen of 70 bees was dissected by peeling off the tergit with forceps in order to expose the honey sac. The honey sac was removed and placed on a microscope slide. In each honey sac content pollen grain types were determined, and the honey sacs containing Mentha spp. pollen grains were frozen. After freezing, the entire content of analyzed honey sacs was pooled and put in a glass tube at $4{ }^{\circ} \mathrm{C}$ until the volatiles isolation.

\subsection{Ultrasonic Solvent Extraction (USE)}

Ultrasound-assisted solvent extraction (USE) was performed in an ultrasound cleaning bath (Elmasonic Typ S $30 \mathrm{H}$, Germany) by the indirect sonication mode (sweep mode), at the frequency of $37 \mathrm{kHz}$ at $25 \pm 3{ }^{\circ} \mathrm{C}$. Forty grams of each Mentha spp. honey sample was dissolved in distilled water $(22 \mathrm{~mL})$ in a $100-\mathrm{mL}$ flask. Magnesium sulfate $(1.5 \mathrm{~g})$ was added and each sample was extensively vortexed. A mixture of pentane-diethyl ether $(1: 2, \mathrm{v} / \mathrm{v})$ and dichloromethane were separately used as the extraction solvents for each honey sample. Sonication was maintained for $30 \mathrm{~min}$. After sonication, the organic layer was separated by centrifugation and filtered over anhydrous $\mathrm{MgSO}$. The aqueous layer was returned to the flask and another batch of the same extraction solvent $(20 \mathrm{~mL})$ was added and extracted by ultrasound for $30 \mathrm{~min}$. The organic layer was separated in the same way as the previous one and filtered over anhydrous $\mathrm{MgSO}_{4}$, and the aqueous layer was sonicated a third time for $30 \mathrm{~min}$ with another batch $(20 \mathrm{~mL})$ of the extraction solvent. Combined organic extracts were concentrated to $0.2 \mathrm{~mL}$ by distillation with a Vigreaux column, and $1 \mu \mathrm{L}$ was used for GC and GC/MS analyses. The content of honey-bee stomach $(35 \mathrm{mg})$ was dissolved in distilled water $(0.5 \mathrm{~mL})$ in a $10 \mathrm{~mL}$ flask, magnesium sulfate $(0.03 \mathrm{mg})$ was added and the sample was extensively vortexed. USE was performed using dichloromethane $(3 \mathrm{~mL})$ applying the same experimental procedure as described for the honey extraction (three-time extraction). 


\subsection{Headspace Solid-Phase Microextraction (HS-SPME)}

The isolation of headspace volatiles was performed using a manual SPME fiber with a layer of polydimethylsiloxane/divinylbenzene (PDMS/DVB) obtained from Supelco Co (Bellefonte, PA, USA). The fiber was conditioned prior to use according to the manufacturer's instructions. For HSSPME extraction, honey/saturated water solution $(5 \mathrm{~mL}, 1: 1 \mathrm{v} / \mathrm{v}$; saturated with $\mathrm{NaCl})$ was placed in a $15 \mathrm{~mL}$ glass vial and hermetically sealed with PTFE/silicone septa. The vial was maintained in a water bath at $60{ }^{\circ} \mathrm{C}$ during equilibration $(15 \mathrm{~min})$ and extraction $(45 \mathrm{~min})$ and was partially submerged so that the liquid phase of the sample was below the water level. All the experiments were performed under constant stirring $(1,000 \mathrm{rpm})$ with a magnetic stirrer. After sampling, the SPME fiber was withdrawn into the needle, removed from the vial, and inserted into the injector $\left(250{ }^{\circ} \mathrm{C}\right)$ of the $\mathrm{GC}$ and GC-MS for 6 min where the extracted volatiles were thermally desorbed directly to the GC column.

\subsection{Gas Chromatography and Mass Spectrometry (GC, GC/MS)}

Gas chromatography analyses were performed on an Agilent Technologies (Palo Alto, CA, USA) gas chromatograph model 7890A equipped with flame ionization detector, mass selective detector, model 5975C and capillary column HP-5MS [(5\%-phenyl)-methylpolysiloxane Agilent J \& W GC column, $30 \mathrm{~m}, 0.25 \mathrm{~mm}$ i.d., coating thickness $0.25 \mu \mathrm{m}$ ]. Chromatographic conditions were as follows: helium was carrier gas at $1 \mathrm{~mL} \cdot \mathrm{min}^{-1}$, injector temperature was $250{ }^{\circ} \mathrm{C}$, and FID detector temperature was $300{ }^{\circ} \mathrm{C}$. HP-5MS column temperature was programmed at $70{ }^{\circ} \mathrm{C}$ isothermal for 2 min, and then increased to $200{ }^{\circ} \mathrm{C}$ at a rate of $3{ }^{\circ} \mathrm{C} \cdot \mathrm{min}^{-1}$ and held isothermal for $18 \mathrm{~min}$. The injected volume was $1 \mu \mathrm{L}$ and the split ratio was 1:50. MS conditions were: ionization voltage $70 \mathrm{eV}$; ion source temperature $230{ }^{\circ} \mathrm{C}$; mass scan range: 30-300 mass units. The analyses were carried out in duplicate.

\subsection{Data Analysis and Data Evaluation}

The individual peaks were identified by comparison of their retention indices (relative to $\mathrm{C}_{9}-\mathrm{C}_{25} n$ alkanes for HP-5MS) to those of authentic samples and literature [33], as well as by comparing their mass spectra with the Wiley 275 MS library (Wiley, New York, USA) and NIST 02 (Gaithersburg, MD, USA) mass spectral databases. The percentage composition of the samples was computed from the GC peak areas using the normalization method (without correction factors). The component percentages (Tables 1 and 2) were calculated as mean values from duplicate GC and GC-MS analyses.

\section{Conclusions}

The chemical constituents of the organic extractives from Mentha spp. honey, headspace and the corresponding bee-stomach revealed the common compounds methyl syringate, vomifoliol and 3,7-dimethylocta-1,5-dien-3,7-diol (terpendiol I) as well as hotrienol that can be related to the plant origin and used for the characterization of this honey. Methyl syringate was the most abundant compound of the honey solvent extractives, followed by vomifoliol. The major honey headspace compounds were hotrienol, cis- and trans-linalool oxides, linalool and neroloxide Comparison of the honey organic extractives with the corresponding bee-stomach indicated that methyl syringate and 
vomofoliol were transferred to the honey, while terpendiol I was partially transformed to hotrienol in ripened honey.

\section{Acknowledgements}

This research has been funded by UKF project 25/08 (Evaluation of Unifloral Honeys - Chemical Fingerprinting and Nutritional Properties) and supported by PIP d.o.o., KONCEPT-MEDIA d.o.o. and GODAX-PRO d.o.o.

\section{References}

1. Castro-Vázquez, L.; Díaz-Maroto, M.C.; González-Viñas, M.A.; Pérez-Coello, M.S. Differentiation of monofloral citrus, rosemary, eucalyptus, lavender, thyme and heather honeys based on volatile composition and sensory descriptive analysis. Food Chem. 2009, 112, 1022-1030.

2. De Maria, C.A.B.; Moreira, R.F.A. Volatile compounds in floral honeys. Quim. Nova 2003, 26, 90-96.

3. Alissandrakis, E.; Tarantilis, P.A.; Harizanis, P.C.; Polissiou, M. Evaluation of four isolation techniques for honey aroma compounds. J. Sci. Food Agric. 2005, 85, 91-97.

4. Alissandrakis, E.; Daferera, D.; Tarantilis, P.A.; Polissiou, M.; Harizanis, P.C. Ultrasound-assisted extraction of volatile compounds from citrus flowers and citrus honey. Food Chem. 2003, 82, $575-582$.

5. D'Arcy, B.R.; Rintoul, G.B.; Rowland, C.Y.; Blackman, A.J. Composition of Australian honey extractives. 1. Norisoprenoids, monoterpenes, and other natural volatiles from blue gum (Eucalyptus leucoxylon) and yellow box (Eucalyptus melliodora) honeys. J. Agric. Food Chem. 1997, 45, 1834-1843.

6. Soria, A.C.; Martínez-Castro, I.; Sanz, J. Analysis of volatile composition of honey by solid phase microextraction and gas chromatography-mass spectrometry. J. Sep. Sci. 2003, 26, 793-801.

7. Bertelli, D.; Papotti, G.; Lolli, M.; Sabatini, A.G.; Plessi, M. Development of an HS-SPME-GC method to determine the methyl anthranilate in Citrus honeys. Food Chem. 2008, 108, 297-303.

8. Castro-Vázquez, L.; Diáz-Maroto, M.C.; Pérez-Coello, M.S. Volatile composition and contribution to the aroma of Spanish honeydew honeys. Identification of a new chemical marker, J. Agric. Food Chem. 2006, 54, 4809-4813.

9. de la Fuente, E.; Valencia-Barrera, R.M.; Martínez-Castro, I.; Sanz, J. Occurrence of 2-hydroxy5-methyl-3-hexanone and 3-hydroxy-5-methyl-2-hexanone as indicators of botanic origin in eucalyptus honeys. Food Chem. 2007, 103, 1176-1180.

10. Guyot, C.; Bouseta, A.; Scheirman, V.; Collin, S. Floral origin markers of chestnut and lime tree honeys. J. Agric. Food Chem. 1998, 46, 625-633.

11. El-Ghorab, A.H. The chemical composition of Mentha pulegium L. essential oil from Egypt and its antioxidant activity. J. Essent. Oil Bear. Plants 2006, 9, 183-195.

12. Mahboubi, M.; Haghi, G. Antimicrobial activity and chemical composition of Mentha pulegium L. essential oil. J. Ethnopharmacol. 2008, 119, 325-327. 
13. Jerković, I.; Mastelić, J. Free and glycosidically bound volatiles composition of Mentha aquatica. Croat. Chem. Acta 2001, 74, 431-439.

14. Celis, M.; Diéz, M.J. Análisis polínico de mieles de La Cabrera baja (Montes de Léon, España). Acta Bot. Malacit. 1995, 20, 91-96.

15. Terrab, A.; Valdés Castrillón, B.; Díez Dapena, M. J. Pollen analysis of honeys from the Gharb region (NW Morocco). Grana 2001, 40, 210-216.

16. Pérez-Arquillué, C.; Ucar Casorran, A.; Herrera Marteache, A; Gómez Ferreras, C. Análisis polínico aplicado a la determinación del origen botánico de mieles de Monegros (España). An. Bromatol. 1988, 40, 265-277.

17. Valencia Barrera, R.M.; Fombella Blanco, M.A.; Fernández González, D. Espectro polínico de mieles de las comarcas leonesas de Omaña, Arguellos y Cepeda. In Trabajos de Palinología básica y aplicada; Mateo, I., Dupré, M., Gümes, J., Burgaz, M.L., Eds.; X Simposio de Palinología (A.P.L.E), Universidad de Valencia: Valencia, Spain, 1994; pp. 111-119.

18. Naef, R.; Jaquier, A.; Velluz, A.; Bachofen, B. From the linden flower to linden honey - volatile constituents of linden nectar, the extract of bee-stomach and ripe honey. Chem. Biodiv. 2004, 1, 1870-1879.

19. Fröhlich, B.; Riederer, M.; Tautz, J. Chemotactic classification of comb and cuticular waxes of the honey bee Apis mellifera carnica. J. Chem. Ecol. 2000, 26, 123-137.

20. Keeling, C.I.; Slessor, K.N.; Higo, H.A.; Winston, M.L. Isolation and identification of new components of the honey bee (Apis mellifera L.) queen retinue pheromone. Proc. Nat. Acad. Sci. USA 2003, 100, 4486-4491.

21. Joerg, E.; Sontag, G. Multichannel coulometric detection coupled with liquid chromatography for determination of phenolic esters in honey. J. Chromatogr. A 1993, 635, 137-142.

22. Tuberoso, C.I.G.; Bifulco, E.; Jerković, I.; Caboni, P.; Cabras, P.; Floris, I. Methyl syringate: a chemical marker of asphodel (Asphodelus microcarpus Salzm. et Viv.) monofloral honey J. Agric. Food Chem. 2009, 57, 3895-3900.

23. Robbins, R.J. Phenolic acids in foods: an overview of analytical methodology. J. Agric. Food Chem. 2003, 51, 2866-2887.

24. D’Abrosca, B.; Della Greca, M.; Fiorentino, A.; Monaco, P.; Zarrelli, A. Low molecular weight phenols from the bioactive aqueous fraction of Cestrum Parqui. J. Agric. Food Chem. 2004, 52, 4101-4108.

25. Leu, Y.-L.; Wang, Y.-L.; Huang, S.-C.; Shi, L.-S. Chemical constituent from roots of Taraxacum formosanum. Chem. Pharm. Bull. 2005, 53, 853-855.

26. Fujimatu, E.; Ishikawa, T.; Kitajima, J. Aromatic compound glucosides, alkyl glucoside and glucide from the fruit of anise. Phytochemistry 2003, 63, 609-616.

27. Borčić, I.; Radonić, A.; Grzunov, K. Comparison of the volatile constituents of propolis gathered in different regions of Croatia. Flav. Fragr. J. 1996, 11, 311-313.

28. Guyot, C.; Scheirman, V.; Collin, S. Floral origin markers of heather honeys: Calluna vulgaris and Erica arborea. Food Chem. 1999, 64, 3-11.

29. Jerković, I.; Tuberoso, C.I.G.; Marijanović, Z.; Jelić, M.; Kasum, A. Headspace, volatile and semi-volatile patterns of Paliurus spina-christi unifloral honey as markers of botanical origin. Food Chem. 2009, 112, 239-245. 
30. Rowland, C.Y.; Blackman, A.J.; D'Arcy, B.; Rintoul, G.B. Comparison of organic extractives found in leatherwood (Eucryphia lucida) honey and leatherwood flowers and leaves. J. Agric. Food Chem. 1995, 43, 753-763.

31. Alissandrakis, E.; Tarantilis, P.A.; Harizanis, P.C.; Polissiou, M. Aroma investigation of unifloral Greek citrus honey using solid-phase microextraction coupled to gas chromatographic-mass spectrometric analysis. Food Chem. 2007, 100, 396-404.

32. Pérez, R.A.; Sánchez-Brunete, C.; Calovo, R.M.; Tadeo, J.L. Analysis of volatiles from Spanish honeys by solid-phase microextraction and gas chromatography-mass spectrometry. J. Agric. Food Chem. 2002, 50, 2633-2637.

33. Louveaux, J.; Maurizio, A.; Vorwohl, G. Methods of melissopalynology. Bee World 1978, 59, 139-153.

34. El-Sayed, A.M. The Pherobase: Database of Insect Pheromones and Semiochemicals. http://www.pherobase.com/, accessed on 22 April 2010.

Sample Availability: Contact the authors.

(C) 2010 by the authors; licensee MDPI, Basel, Switzerland. This article is an open-access article distributed under the terms and conditions of the Creative Commons Attribution license (http://creativecommons.org/licenses/by/3.0/). 